\title{
Birth month, birth season, and overall and cardiovascular disease mortality in US women: prospective cohort study
}

Check for updates
${ }^{1}$ Channing Division of Network

Medicine, Brigham and

Women's Hospital and Harvard

Medical School, Boston, MA

02115, USA

${ }^{2}$ Department of Epidemiology, Harvard T H Chan School of Public Health, Boston, MA, USA

${ }^{3}$ Department of Epidemiology, Centre for Public Health,

Medical University of Vienna, Vienna, Austria

Correspondence to:

ESSchernhammer

eva.schernhammer@channing. harvard.edu

(ORCID 0000-0002-4337-9415)

Additional material is published online only. To view please visit the journal online.

Cite this as: BMJ 2019;367:16058 http://dx.doi.org/10.1136/bmj.16058

Accepted: 22 August 2019

\author{
Yin Zhang, ${ }^{1,2}$ Elizabeth E Devore, ${ }^{1}$ Susanne Strohmaier, ${ }^{1}$ Francine Grodstein, ${ }^{1}$ \\ Eva S Schernhammer ${ }^{1,2,3}$
}

\section{ABSTRACT}

\section{OBJECTIVES}

To evaluate the associations between birth month, birth season, and overall and cardiovascular disease mortality, and to examine the role of familial and socioeconomic factors in these associations.

DESIGN

Prospective cohort study.

SETTING

Nurses' Health Study, established in 1976, an ongoing prospective cohort study in the United States.

\section{PARTICIPANTS}

Female registered nurses who reported information on date of birth at study enrolment $(n=116911,1976$ 2014 , followed for 38 years).

\section{EXPOSURE}

Birth month and astronomical birth season (based on solstices and equinoxes as boundaries of the season categories).

\section{MAIN OUTCOME MEASURES}

Age and various multivariable adjusted hazard ratios and $95 \%$ confidence intervals for the association between birth months (using November as the reference), astronomical birth season (using autumn as the reference), and overall and cardiovascular disease specific mortality were assessed using Cox proportional hazards models.

RESULTS

Among study participants, 43248 overall deaths were documented during 4136364 person years of follow- up since enrolment, including 8360 cardiovascular disease related deaths. In fully adjusted multivariable analyses, no significant association was observed between birth month, birth season, and overall mortality. Compared with women born in November, increased cardiovascular disease mortality was observed among those born from March to July (hazard ratio for March, 1.09, 95\% confidence interval 0.98 to 1.21 ; April, $1.12,1.00$ to 1.24; May, 1.08, 0.98 to 1.20 ; June, $1.07,0.96$ to 1.19 ; and July 1.08 , 0.98 to 1.20). Those born in April had the highest cardiovascular disease mortality, and those born in December had the lowest (December, 0.95, 0.85 to 1.06$)$. The relative difference between the lowest and highest risk month was $17.89 \%$. Women born in spring $(1.10,1.04$ to 1.17$)$ and summer $(1.09,1.03$ to 1.16) had a higher cardiovascular disease mortality than women born in the autumn. Adjustment for familial and socioeconomic factors did not change these results. The relative difference between the lowest and highest risk season was $10.00 \%$.

CONCLUSION

Participants born in the spring and summer (especially those born in March-July) had a slight but significant increase in cardiovascular disease specific mortality. However, no seasonal birth month effect was observed among women for overall mortality. Familial and socioeconomic factors did not appear to alter these associations. Further studies are required to confirm these findings and reveal mechanisms of these seasonal birth month effects in cardiovascular disease mortality.

\section{Introduction}

The well known fetal origins hypothesis ${ }^{12}$ and developmental origins of health and disease concept ${ }^{3}$ have associated early life exposures with the development of adverse health outcomes in adulthood. Season and month of birth represent well defined and frequently used proxies for various environmental and other factors in prenatal and early postnatal life. Previous studies have identified associations between season and month of birth and overall and cardiovascular disease related mortality in the United States, ${ }^{45}$ Sweden, ${ }^{6}$ Germany, ${ }^{89}$ Austria, ${ }^{10} 11$ Denmark, ${ }^{10}{ }^{11}$ Australia, $^{10}$ Lithuania, ${ }^{12}$ Japan, ${ }^{13}$ and Spain. ${ }^{14}$ Results from the northern hemisphere show relatively consistently that individuals born in November have the lowest risk of overall and cardiovascular mortality, whereas individuals born in the spring or summer (peaking in May) have the highest mortality risk..$^{4-13}$ By contrast, in the southern hemisphere, the pattern of seasonal birth month effects on mortality seems to be shifted by half a year. ${ }^{10}$ 
Mechanisms driving these observed associations remain inconclusive, with prenatal and early postnatal exposures including seasonal fluctuations in nutrition availability $^{11015-21}$ (eg, intake of fruits, vegetables, and total calories), infections and inflammatory causes, ${ }^{21-23}$ climatic temperature and air pollution, ${ }^{24-27}$ and sunlight (maternal vitamin D levels, and solar cycles) ${ }^{25}$ 28-30 having been suggested. Familial and socioeconomic factors have been questioned as potential confounders. ${ }^{4}{ }^{31-34}$ Near-birth familial and socioeconomic factors could influence the timing of conception and thus birth-for example, those whose parents have more favorable factors might choose to give birth avoiding colder months. ${ }^{41-33}$ Later life familial and socioeconomic factors might also be associated with overall and cardiovascular mortality in adulthood. ${ }^{34}$ However, to our knowledge, none of these previous studies has been able to adequately control for these factors and other potential confounders, ${ }^{4-14}$ limiting the interpretability of their findings. ${ }^{235}$

With detailed documentation of demographics, lifestyle, medical history, anthropometric data, and familial and socioeconomic factors, the Nurses' Health Study longitudinal cohort in the $\mathrm{US}^{36}$ offers an unparalleled resource to perform a large scale prospective study to add to existing evidence and overcome some limitations of previous studies. We thus examined the association between seasonal birth month and overall and cardiovascular mortality in the large Nurses' Health Study cohort, exploring the role of familial and socioeconomic factors in this association in detail.

\section{Methods}

\section{Study population}

We conducted the present analysis using data from the Nurses' Health Study cohort, the details of which have been reported previously. ${ }^{36-38}$ In brief, it is an ongoing large prospective cohort study conceived in the early 1970s and initiated in 1976, with 121700 US female registered nurses aged 30 to 55 years involved at enrolment. Demographic information was collected from initial questionnaires. By mailing self administered questionnaires to all participants biennially throughout follow-up, information on lifestyle characteristics, medical history, and newly diagnosed diseases were regularly updated, with a cumulative follow-up rate of greater than 90\% of potential person time having been achieved. Information on familial and socioeconomic factors and other factors of interest was also collected. We excluded participants who were diagnosed with any cardiovascular disease before or at baseline, or who had no information on birthdate. Women who reported a history of having been born prematurely were also excluded from analyses.

\section{Ascertainment of deaths}

Deaths were ascertained through the National Death Index and next-of-kin or postal authority reporting, with an identifying rate of more than $96 \%$ in the Nurses' Health Study cohort. ${ }^{39} 40$ Cohort investigators reviewed death certificates, and requested permission from next of kin of dead participants to review their medical records. Causes of death were then assigned according to the ICD-8 (international classification of diseases, 8th revision). We specifically considered overall mortality and cardiovascular disease related deaths (ICD-8 codes 390.0 to 458.9 and 795.0 to 795.9) in this analysis.

\section{Ascertainment of birth month and astronomical birth season}

Participants reported information on their exact birth date in the Nurses' Health Study cohort on the 1976 questionnaire. According to their responses, month and season of birth were then ascertained. We used astronomical birth season in our study, based on solstices and equinoxes as the bounds of the season categories. In view of minor variations on the timings of solstices and equinoxes in different years, four astronomical birth seasons were created following the standard definition of spring (March 21 to June 20), summer (June 21 to September 20), autumn (September 21 to December 20), and winter (December 21 to March 20).

\section{Ascertainment of covariates}

Covariates included age, race, body mass index, smoking status, physical activity, and history of hypertension, hypercholesterolemia, and type 2 diabetes. We further considered near-birth and other familial and socioeconomic indicators as potential confounders. Information on race and body weight was captured on the initial questionnaire in 1976. Detailed information on familial and socioeconomic factors was then assessed, including the nurses' husbands' educational level (reported in 1992), as well as their parents' home ownership at birth of the respective nurse (reported in 2004), parents' occupation in childhood (reported in 1976), and their mothers' smoking status during pregnancy (reported in 2004). Via the regular biennial questionnaires throughout follow-up, participants subsequently reported and updated anthropometric data (self reported body weight, which was used to calculate participants' body mass index with height), lifestyle factors (self reported smoking status), and medical history (self reported diagnosis of diseases, then verified by study physicians who reviewed medical records, including hypertension, hypercholesterolemia, and type 2 diabetes). Participants also received questionnaires quadrennially for reporting their frequency of engaging in common recreational activities, based on which total physical activity in metabolic equivalent tasks per week was then calculated. The validity and reproducibility of information on anthropometric data, health related behaviors, and major chronic diseases have been previously described in detail. ${ }^{41-43}$

\section{Statistical analysis}

We analyzed month and season of birth in relation to overall and cardiovascular mortality. To investigate potential heterogeneity across major cardiovascular 
disease subtypes, we further performed subtype analysis-that is, separate analyses for ischemic heart disease and cerebrovascular disease across birth season. We were unable to consider analyses examining birth season in relation to other cardiovascular disease subtypes, or analyses for any of the cardiovascular disease subtypes across birth months, because of power limitations.

Person years of follow-up were calculated from the date of return of the initial questionnaire in 1976 until the date of death recorded, loss to follow-up, or the end of follow-up (the date of follow-up completion was defined as 30 June 2014 in our present study), whichever was earliest. Age and multivariable adjusted hazard ratios and 95\% confidence intervals for overall and cardiovascular disease specific mortality were estimated by using Cox proportional hazards regression models conditioning on age (in 5-year categories) and questionnaire cycle (2-year intervals), across birth months (using November as the reference group) and astronomical birth seasons (using autumn as the reference group).

We controlled for the following covariates in multivariable models: age (in 5-year categories; $\leq 55$, 56-60, 61-65, 66-70, 71-75, >75), race (white, black, other), body mass index (<21.0, 21.0-22.9, 23.0$24.9,25.0-27.4,27.5-29.9,30.0-34.9$, and $\geq 35.0$, implementing a 4-year lag time to account for potential reverse causation), smoking status (never smoker, past smoker, current smoker $<25$ cigarettes/day, current smoker $\geq 25$ cigarettes/day), physical activity (metabolic equivalent tasks/week, in fifths), husbands' educational level (less than high school, some high school, high school graduate, college, graduate school), history of hypertension (yes, no), history of hypercholesterolemia (yes, no, with 4-year lag time), and history of type 2 diabetes (yes, no).

To facilitate better control of confounding and further investigate the effect of familial and socioeconomic factors in the associations between birth month, birth season, and overall and cardiovascular disease specific mortality, we also controlled for these factors of the participants around the time they were born. These factors included parental home ownership at birth (yes, no), father's occupation in childhood (professional/executive, sales/clerical, craftsman/ service, laborer/farmer/military, stay-at-home parent), mother's occupation in childhood (professional/ executive, sales/clerical, craftsman/service, laborer/ farmer/military, stay-at-home parent), and mother's smoking status during pregnancy (yes, no).

In all, we performed four models in this study that adjusted for age, for age and familial and socioeconomic factors, for age and factors other than familial and socioeconomic factors, and for all covariates. Information on repeatedly measured covariates, including participants' lifestyle factors, medical history, and anthropometric data, was updated in models biennially throughout follow-up. To minimize missing information on these time-varying variables, missing values were carried forward from the latest valid data in previous follow-up cycles, and missing indicators were included in the models for the remaining covariates with no values after replacement. Analyses were performed by SAS software (version 9.4 for UNIX; SAS Institute, Cary, NC), all tests were two sided, and $\mathrm{P}$ values less than 0.05 were considered statistically significant.

\section{Patient and public involvement}

Participants were not involved in setting the research question or the outcome measures, nor were they involved in the design or implementation of the study. No participants were asked to advise on interpretation or writing up of the manuscript. The participants are updated on findings and developments of the Nurses' Health Study cohort through annual newsletters and the official website (https://www.nurseshealthstudy.org).

\section{Results}

\section{Population characteristics}

A total of 116911 women participating in Nurses' Health Study were eligible for inclusion in our study. During 38 years of follow-up (1976-2014) and 4136364 person years since enrolment, 43248 overall deaths were documented, including 8360 cardiovascular disease specific death. In addition to cardiovascular disease, main causes of death included cancer $(n=13954)$ and other causes $(n=20934)$ including neurodegenerative disease $(\mathrm{n}=4318)$, respiratory disease $(n=2879)$, or infections $(n=1811)$. Among all the documented cases of death, the mean age at death was 75.5. Mean age at death varied between participants who died from cardiovascular disease (74.6, similar to that of all cause deaths), cancer (69.8), neurodegenerative disease (82.3), respiratory disease (76.8), and infections (71.3). Nearbirth characteristics and other characteristics of the study population at enrolment in 1976 are presented in table 1 . Women born in the winter were more likely to have an increased adult body mass index. No other major variation was observed across birth season in any of these characteristics.

\section{Birth month, birth season, and overall mortality}

Table 2 presents age adjusted and multivariable adjusted hazard ratios and 95\% confidence intervals of overall death among study participants across birth months and astronomical birth seasons. Overall, no significant association was seen between birth month or astronomical birth season and overall mortality in age adjusted analyses (table 2, model 1), or in any of the multivariable analyses, after additionally adjusting for familial and socioeconomic factors (model 2), factors other than familial and socioeconomic factors (model 3), and all covariates (model 4).

\section{Birth month, birth season, and cardiovascular disease mortality}

Age adjusted and multivariable adjusted hazard ratios and $95 \%$ confidence intervals of cardiovascular death among the study population across birth months and 


\begin{tabular}{|c|c|c|c|c|}
\hline Characteristic & Winter $(n=28$ 196) & Spring $(n=28596)$ & Summer $(n=30490)$ & Autumn $(n=29629)$ \\
\hline \multicolumn{5}{|l|}{ Birth year } \\
\hline$<1925$ & $4036(14.31)$ & $4118(14.40)$ & 4569 (14.99) & $4512(15.23)$ \\
\hline $1925-29$ & $5885(20.87)$ & $5934(20.75)$ & $6253(20.51)$ & $5951(20.09)$ \\
\hline $1930-34$ & $5843(20.72)$ & $5935(20.75)$ & $6242(20.47)$ & $6142(20.73)$ \\
\hline $1935-39$ & $5704(20.23)$ & $5914(20.68)$ & $6178(20.26)$ & $5730(19.34)$ \\
\hline $1940-44$ & $6728(23.86)$ & $6695(23.41)$ & $7248(23.77)$ & $7294(24.62)$ \\
\hline \multicolumn{5}{|l|}{ Race } \\
\hline White & $27245(96.63)$ & 27677 (96.79) & $29518(96.81)$ & $28645(96.68)$ \\
\hline Black & $671(2.38)$ & $643(2.25)$ & $659(2.16)$ & $672(2.27)$ \\
\hline Other & $280(0.99)$ & $276(0.97)$ & $313(1.03)$ & $312(1.05)$ \\
\hline \multicolumn{5}{|l|}{ Body mass index } \\
\hline$<20$ & $3441(12.33)$ & $3512(12.40)$ & 3916 (12.98) & $3863(13.17)$ \\
\hline 20 to $<22$ & $7516(26.93)$ & $7791(27.51)$ & $8390(27.81)$ & $8077(27.53)$ \\
\hline 22 to $<25$ & $8940(32.03)$ & $8913(31.47)$ & $9389(31.12)$ & $9177(31.28)$ \\
\hline 25 to $<30$ & $5691(20.39)$ & $5749(20.30)$ & $6012(19.93)$ & $5866(20.00)$ \\
\hline$\geq 30$ & $2322(8.32)$ & $2355(8.32)$ & $2465(8.17)$ & $2353(8.02)$ \\
\hline \multicolumn{5}{|l|}{ Smoking status } \\
\hline Never smoked & $12170(43.28)$ & $12453(43.67)$ & $13252(43.61)$ & $12673(42.89)$ \\
\hline Past smoker & $6559(23.33)$ & $6677(23.41)$ & $7059(23.23)$ & $6924(23.43)$ \\
\hline Current smoker & $9391(33.40)$ & $9387(32.92)$ & $10080(33.17)$ & 9951 (33.68) \\
\hline \multicolumn{5}{|c|}{ Physical activity (metabolic equivalent tasks/week) } \\
\hline$<3$ & $5382(28.35)$ & $5384(27.72)$ & $5895(28.42)$ & $5839(29.04)$ \\
\hline 3 to $<9$ & $5213(27.46)$ & $5175(26.64)$ & $5562(26.81)$ & $5309(26.40)$ \\
\hline 9 to $<18$ & $3542(18.66)$ & $3826(19.70)$ & $4018(19.37)$ & 3814 (18.97) \\
\hline 18 to $<27$ & $1977(10.41)$ & $2093(10.78)$ & $2199(10.60)$ & $2179(10.84)$ \\
\hline 27 to $<42$ & $1579(8.32)$ & $1663(8.56)$ & $1703(8.21)$ & $1645(8.18)$ \\
\hline$\geq 42$ & $1290(6.80)$ & $1283(6.61)$ & $1366(6.59)$ & $1323(6.58)$ \\
\hline Hypertension & $3253(11.54)$ & $3426(11.98)$ & $3431(11.25)$ & $3325(11.22)$ \\
\hline Hypercholesterolemia & $940(3.33)$ & $966(3.38)$ & $1020(3.35)$ & $938(3.17)$ \\
\hline Type 2 diabetes & $517(1.83)$ & $520(1.82)$ & $526(1.73)$ & $534(1.80)$ \\
\hline \multicolumn{5}{|l|}{ Husband's education } \\
\hline High school & $7942(46.89)$ & $8174(46.88)$ & $8481(45.98)$ & $8302(45.82)$ \\
\hline College grad & $4964(29.31)$ & $4984(28.58)$ & $5464(29.62)$ & $5445(30.05)$ \\
\hline Grad school & $4030(23.80)$ & $4279(24.54)$ & $4500(24.40)$ & $4372(24.13)$ \\
\hline Parents owned home at birth & $7517(44.33)$ & 7607 (43.89) & $8081(43.97)$ & 7995 (44.19) \\
\hline \multicolumn{5}{|l|}{ Mother's occupation } \\
\hline Professional/executive & $2486(9.35)$ & $2354(8.72)$ & $2520(8.76)$ & $2498(8.92)$ \\
\hline Sales/clerical & $2836(10.66)$ & $3045(11.29)$ & $3263(11.34)$ & $3274(11.69)$ \\
\hline Craftsman/service & $2806(10.55)$ & $2879(10.67)$ & $3140(10.91)$ & $3028(10.81)$ \\
\hline Labor/farmer/military & $144(0.54)$ & $132(0.49)$ & $149(0.52)$ & $150(0.54)$ \\
\hline Stay at home & $18324(68.90)$ & $18572(68.83)$ & $19704(68.47)$ & $19067(68.06)$ \\
\hline \multicolumn{5}{|l|}{ Father's occupation } \\
\hline Professional/executive & $6971(27.48)$ & $7251(28.15)$ & $7661(27.91)$ & $7530(28.10)$ \\
\hline Sales/clerical & $9271(36.54)$ & $9465(36.75)$ & $10144(36.95)$ & $9849(36.75)$ \\
\hline Craftsman/service & $5661(22.31)$ & $5716(22.19)$ & $6112(22.27)$ & $6063(22.62)$ \\
\hline Labor/farmer/military & $3432(13.53)$ & $3296(12.80)$ & $3497(12.74)$ & $3328(12.42)$ \\
\hline Stay at home & $36(0.14)$ & $29(0.11)$ & $37(0.13)$ & $30(0.11)$ \\
\hline Mother smoked during pregnancy & $1689(11.27)$ & $1783(11.65)$ & $1962(12.11)$ & 1909 (11.99) \\
\hline
\end{tabular}

astronomical birth seasons are shown in table 3. In age adjusted analyses (table 3, model 1), compared with women born in November, those born from March to July had a higher mortality for cardiovascular disease (hazard ratio for March, 1.08, 95\% confidence interval 0.97 to 1.20; April, 1.11, 1.00 to 1.23; May, 1.08, 0.97 to 1.20 ; June, $1.06,0.95$ to 1.18 ; July, 1.09 , 0.99 to 1.21 ), while women born in December (0.94, 0.84 to 1.05 ) had the lowest cardiovascular disease related mortality. In terms of birth seasons, higher cardiovascular disease related mortality was observed among women born in the spring and summer (hazard ratio for spring, 1.09, 1.03 to 1.16; summer, 1.10, 1.03 to 1.17) compared with those women who born in the autumn.

These positive associations by season and month of birth remained after additionally controlling for familial and socioeconomic factors (table 3, model 2), factors other than familial and socioeconomic factors(table 3, model 3), and all covariates (table 3, model 4), respectively. No major attenuation of the associations was observed after any of these additional adjustments were conducted. In fully adjusted multivariable analyses (table 3, model 4), compared with women born in November, higher cardiovascular mortality was observed among those born from March to July (hazard 


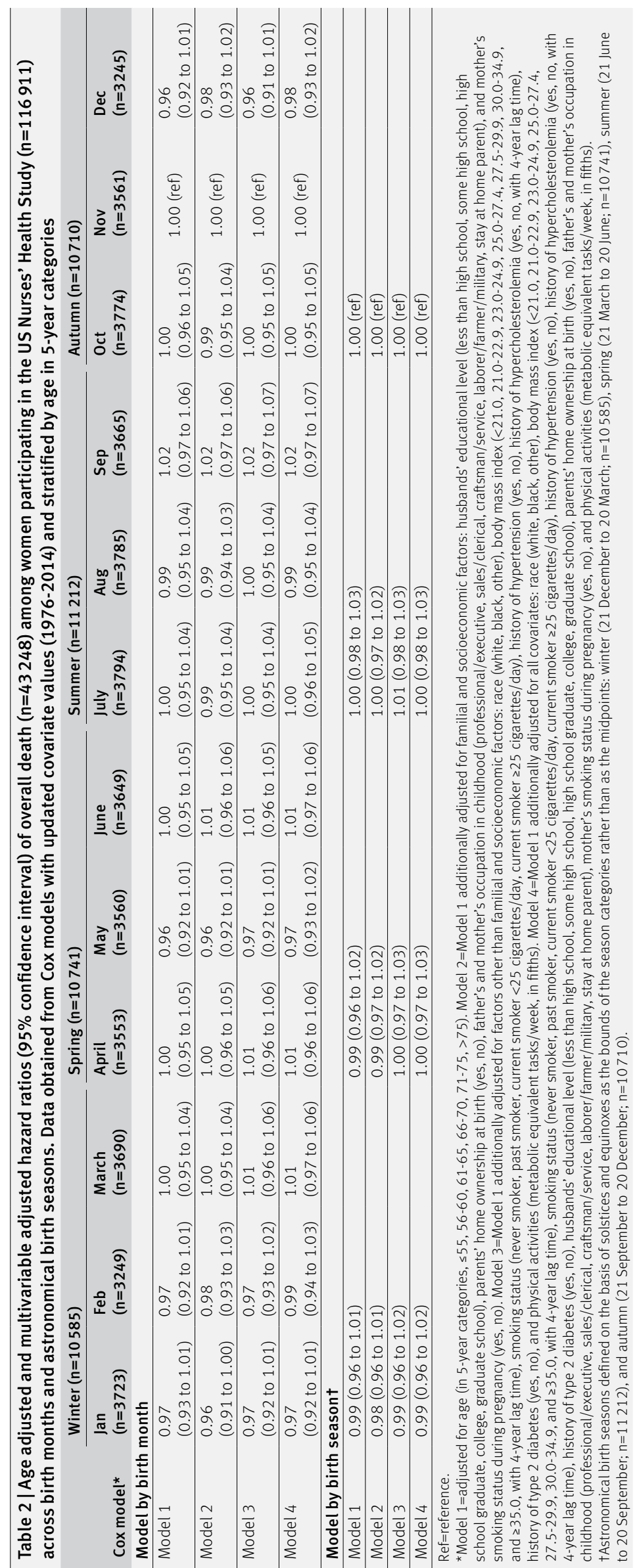

ratio for March, 1.09, 95\% confidence interval 0.98 to 1.21; April, 1.12, 1.00 to 1.24; May, 1.08, 0.98 to 1.20; June $1.07,0.96$ to 1.19 ; July, $1.08,0.98$ to 1.20 ), with the highest cardiovascular mortality for those born in April, and lowest among those born in December $(0.95,0.85$ to 1.06$)$. Women born in the spring and summer had higher cardiovascular mortality (hazard ratio for spring, $1.10,1.04$ to 1.17 ; summer, 1.09, 1.03 to 1.16$)$ than those born in autumn. The relative difference between the lowest and highest risk month was $17.89 \%$, and this difference between the lowest and highest risk season was $10.00 \%$.

In fully adjusted multivariable analyses of the two major subtypes of cardiovascular disease, although with some heterogeneity, both ischemic heart disease specific mortality (hazard ratio for spring, 1.07, 95\% confidence interval 0.96 to 1.18 ; summer, $1.08,0.98$ to 1.20 ; winter $1.10,1.00$ to 1.22 ) and cerebrovascular disease specific mortality $(1.15,1.03$ to $1.30 ; 1.05$, 0.93 to $1.18 ; 0.98,0.87$ to 1.11 ) meaningfully differed between birth season groups, compared with women born in the autumn.

\section{Discussion}

In this large prospective US cohort study, we observed that women born in the spring and summer (especially those born from March through July) had an increased cardiovascular mortality, but not overall mortality, compared with those born in the autumn.

\section{Comparison with other studies}

Prior evidence from the US, ${ }^{45}$ Sweden, ${ }^{6}{ }^{7}$ Germany, ${ }^{8}$ Austria, $^{10} 11$ Denmark, ${ }^{10}{ }^{11}$ Australia, ${ }^{10}$ Lithuania, ${ }^{12}$ Japan, ${ }^{13}$ and Spain ${ }^{14}$ has related seasonal birth month effect to all cause and cardiovascular mortality in later life. Consistent with our current findings, two large longitudinal studies in Sweden (including over six million and four million people, respectively) with 20 years follow-up, reported the lowest cardiovascular mortality among participants born in November. ${ }^{67}$ Individuals born in the spring or summer had the highest overall mortality (peaking in April/May), ${ }^{6}$ and those born from January to August had highest cardiovascular mortality (peaking in March/April). ${ }^{7}$ A US cohort with a similar follow-up time, although distinctively smaller than the Swedish studies and ours, indicated similar findings. ${ }^{4}$ Records from 1.37 million people in a Danish study with 30 years of follow-up (1.17 million deaths were documented) and deaths records of 680000 people in Austria also showed that individuals born in spring (April-June) had a shorter lifespan than those born in autumn (OctoberDecember). ${ }^{1011}$ A cross sectional observation of more than six million deaths in Germany suggested that people born around May had the highest all cause and cardiovascular mortality, while the lowest risks for both types of death were observed among individuals born in November. ${ }^{8}$ Other smaller studies from the US, ${ }^{5}$ Germany, ${ }^{9}$ Lithuania, ${ }^{12}$ and $\operatorname{Japan}^{13}$ also showed similar findings on overall ${ }^{5913}$ and cardiovascular mortality. ${ }^{12}$

Previous epidemiological studies have relatively consistently described individuals born in November 


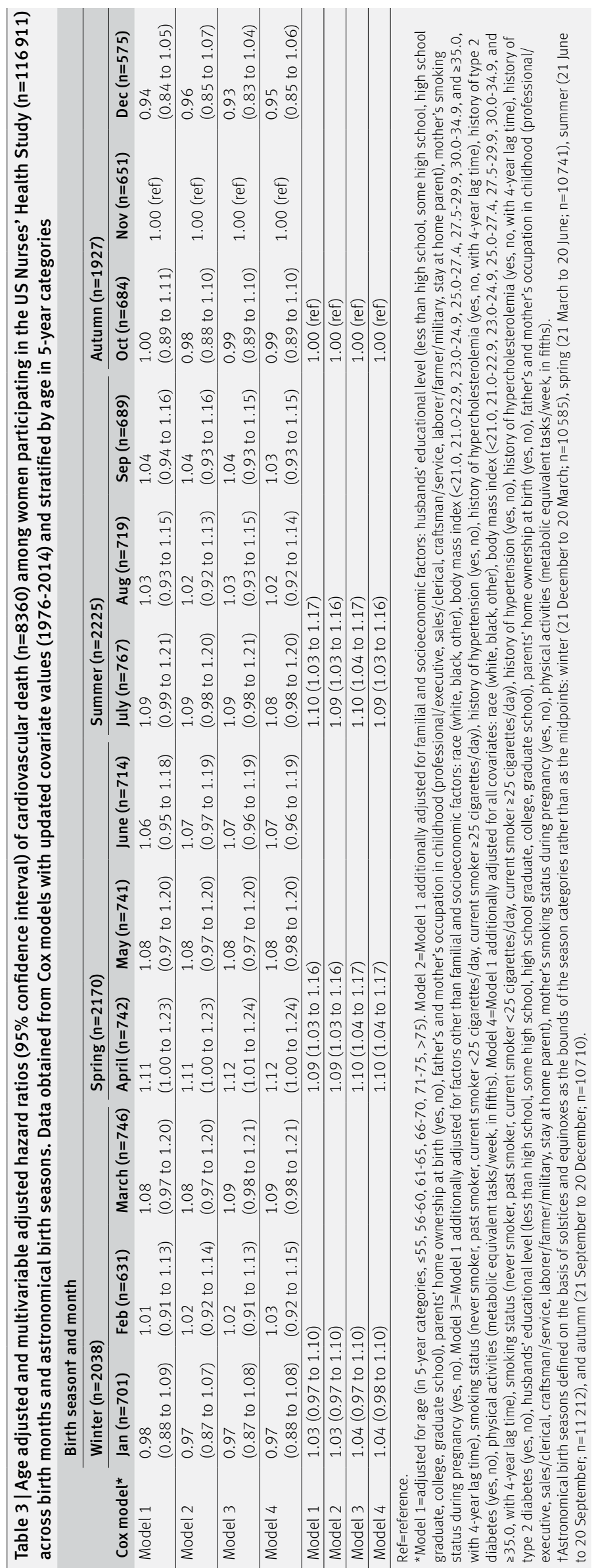

to have the lowest risk of overall and cardiovascular mortality, and those born in the spring or summer to have the highest mortality risk. ${ }^{4-13}$ However, a major concern is that these findings, in support of the fetal origins hypothesis ${ }^{12}$ and developmental origins of health and disease concept, ${ }^{3}$ might be hampered by incomplete and incorrect statistical interpretation (eg, inadequate control for early and later life predictors of mortality, which might both significantly contribute to variation in lifetime health conditions). ${ }^{2} 35$ In particular, a major limitation of these observations ${ }^{4-14}$ is that they have not been able to adequately control for familial and socioeconomic factors-a potentially important confounder-and thus might have rendered these studies vulnerable to bias.

Infact, USdatahaveshown thatnear-birthfamilialand socioeconomic factors could have a role in determining the timing of conception and thus birth, in that women with more favorable familial and socioeconomic factors chose to give birth avoiding colder months. ${ }^{431}$ Swedish and Czech studies reported similar supportive findings that those individuals with unfavorable nearbirth familial and socioeconomic factors were more likely to be born in in November and December. ${ }^{32} 33$ This hypothesis presents an important complement to existing explanations, and near-birth indicators of familial and socioeconomic factors should therefore be conjectured as potential confounders. ${ }^{4} 13$ 31-33 Moreover, a prospective study from Scotland also indicated that familial and socioeconomic factors over a lifetime (including familial and socioeconomic factors near birth as well as in later life) could be associated with overall and cardiovascular mortality. ${ }^{34}$ Here, we present a prospective cohort study with adequate adjustment of potential confounders to evaluate the associations between seasonal birth month and overall and cardiovascular mortality, and to examine the role of familial and socioeconomic factors in these associations in detail.

Cardiovascular disease has remained the leading cause of death in the US. ${ }^{44}$ Our study among US women corroborates the previously observed associations between seasonal birth month and cardiovascular mortality. ${ }^{7} 812$ Compared with US women born in November, we observed higher cardiovascular mortality among those born from March to July (peaking in April), and the lowest among those born in December. Accumulating evidence from the US, ${ }^{45} 46$ Britain, ${ }^{26}$ and Canada $^{47}$ has shown a similar link between season and month of birth and susceptibility to cardiovascular disease in later life. A recent study of more than 1.7 million US individuals indicated the lowest risk of cardiovascular disease observed among those born in the autumn, and indicated the highest risk among those born in the winter (January-March) and spring (April-June), ${ }^{46}$ the trend of which roughly paralleled our finding on cardiovascular mortality. Moreover, certain prenatal and perinatal exposures (eg, nutrition availability, ${ }^{19}$ 48-50 climatic temperature, ${ }^{26}$ sunlight exposure, ${ }^{29}$ and infectious diseases ${ }^{51}$ ) involved in explanations for seasonal birth month effect have been specifically associated with 
variation of cardiovascular outcomes, including familial and socioeconomic factors in early life. ${ }^{52}$

Our finding conflicts with previous studies on overall mortality. ${ }^{4-11} 13$ We observed an association specifically for cardiovascular mortality, but not for overall mortality. However, cardiovascular mortality accounts for only $19.33 \%$ of overall mortality in our study. This fact suggests a specific seasonal birth month effect on cardiovascular disease, rather than confounding, and might not have been observed in previous studies because their cardiovascular mortality accounted for a greater proportion (39.5-46.6\%) of overall mortality than ours. ${ }^{48}$ Collectively, our study provides evidence indicating that the association of fetal and early life factors with cardiovascular disease related mortality might not all be related to an individual's early environment generally tracking on a similar trajectory throughout life, but related to a small but real seasonal effect on later life. The reason behind no significant association being observed between seasonal birth month and overall mortality in our study remains unclear. Maternal vitamin D status during pregnancy, as a correlate of season and month of birth season, ${ }^{53}$ has been described as a suggestive or probable factor affecting adult cardiovascular risk, ${ }^{29}$ indicating one of the initial but provocative clues regarding potential mechanisms.

Season and month of birth represent proxies for various complex environmental and other factors (eg, familial and socioeconomic factors) in prenatal and early postnatal life. The impact and mechanisms of a seasonal birth month effect on overall and cardiovascular mortality might vary across different country specific settings. In our analyses, multivariable adjustment for a range of factors, including detailed assessments of familial and socioeconomic factors around birth and later in life, did not appear to alter the seasonal birth month effect on both overall and cardiovascular mortality, indicating that the association we observed in US women is unlikely to be related to familial and socioeconomic factors. Death records of 220000 native born Australians and 43000 British born Australians showed that the pattern of their seasonal birth month effect on overall mortality mirrored a reversal of that in northern hemisphere countries (Austria and Denmark, shifted by half a year). ${ }^{10}$ In the same study, British born Australians resembled the overall mortality pattern of the northern hemisphere, and were significantly different from native born Australians. ${ }^{10}$ Such findings indicate that in these countries, both climate pattern and familial and socioeconomic factors near birth and in later life might have roles in seasonal birth month effect. However, in another observation of over six million deaths in Germany, stratifying by regions with various socioeconomic status (eg, urban areas/rural areas/ core cities, and different federal states) did not change results, ${ }^{8}$ although Germany might have less variation in familial and socioeconomic factors among their citizens than other countries worldwide.

The impact and mechanism of seasonal birth month effect on mortality might also vary across different time specific settings. Substantial reductions in overall mortality have been witnessed since 1950, which has contributed to a subsequent remarkable increase in lifespan. ${ }^{35}$ In previous observations, study populations were born in the first half or middle of the 20th century or even earlier. This birth period might be important because in most of the countries, seasonality of nutrition availability or infections, for example, are currently not as manifest as they have been in the past. Meanwhile, over the past half century, more seasonal fluctuations in climatic temperature have been observed. In addition, country specific social and economic status has also changed. For instance, results from the earliest observation of Spanish villagers born between 1634 and $1870^{14}$ conflict greatly with findings from study populations born in the 20th century. ${ }^{4-13}$ In more recent cohorts, attenuated seasonal birth month effects in adult lifespan have been observed over time, and might be attributed to these complex changes. ${ }^{10}$ Whether the association between seasonal birth month effect and overall and cardiovascular mortality will still be present or change for people born today remains to be further examined. However, as it stands, findings from previous studies should be interpreted according to both country and time specific settings.

We also found in our study that adult body mass index is higher among US women born in the winter, the mechanisms of which remain to be elucidated. Previous evidence regarding the relation between birth season and adult body mass index remains inconclusive. Our study conflicts with the reported slight increase in adult body mass index among Chinese adults born in the spring and early summer, ${ }^{54}$ and is also inconsistent with the null finding reported in UK populations. ${ }^{55}$ Substantial variability by geographical region (eg, latitudes), near birth environment, familial and socioeconomic factors, and time specific settings could exist. ${ }^{5455}$

\section{Strengths and limitations of study}

Our population based prospective study comprises observations from more than 110000 eligible participants from the US, examining the seasonal birth month effect in overall and cardiovascular mortality. This analysis had several major strengths. Firstly, the nature of a prospective cohort study design limits the potential for recall bias. Secondly, the large number of deaths (43248 overall deaths, including 8360 cardiovascular deaths), long follow-up (38 years), and high follow-up response rates attained $(>90 \%)$, in conjunction with a standardized review of medical records and death certificates by study physicians, provides an unparalleled dataset for us to examine the associations between our exposures and outcomes. In addition, with detailed documentation of demographics and familial and socioeconomic factors of our study population, and repeated biennial assessments of a wide spectrum of information on validated anthropometric indicators, lifestyle factors, and medical history, the Nurses' Health Study offers high quality resources to perform analyses, allowing 
rigorous adjustment for potential confounders that have not been sufficiently controlled in previous studies. The fact that all participants in our study were trained health professionals helps to reduce heterogeneity and thus presents another advantage in minimizing confounding and enhancing the internal validity.

Our study also had several limitations. Firstly, although we conducted extensive multivariate analyses to assess any plausible confounding, residual confounding from potential measurement error in the covariates or other unmeasured confounding cannot be completely ruled out, and causal inference might thus be hindered. Secondly, our study population was not a random sample of US women. All participants were healthcare professionals predominantly with European ancestry (>96\%). Some minority groups in the US, including African Americans, Asian Americans, and Hispanics, were under-represented in our cohort. Although a range of factors, including familial and socioeconomic factors, were carefully controlled in our study, the professional, racial, and ethnic homogeneity might still preclude generalizability of current findings to other demographic groups. Additional investigations are warranted among other diverse populations.

Thirdly, another potential bias, inevitable in all prospective cohort studies, is a differential exposure and outcome experience for participants lost to followup. However, an overall response rate of more than 90\% had been achieved in Nurses' Health Study cohort throughout follow-up, which helped us minimize such bias in this study. Moreover, deaths were ascertained through the National Death Index and next-of-kin or postal authority reporting, with an identifying rate of more than $96 \%$ in the Nurses' Health Study cohort. ${ }^{3940}$ Fourthly, data regarding covariates were unavailable in a certain time (from participants' birth to the initiation of the Nurses' Health Study cohort) because of the specificity of our exposures in this study, presenting another limitation. Information regarding more granular occupational data, and types of seasonal or shift work during parents' fertility or pregnancy timing and child rearing practices, were also not available in this cohort. Finally, possible mechanisms of seasonal birth month effect in mortality remain to be uncovered. Our findings need to be validated by further investigations, and results should be interpreted cautiously in the light of mechanistic, animal, and epidemiological evidence.

\section{Conclusion and public health implications}

This prospective large cohort study suggests that US women born in the spring and summer (especially from March through July) have increased cardiovascular mortality compared with those born in the autumn. Adjustment for a range of factors, including familial and socioeconomic factors around birth and later in life, does not appear to alter these associations. Collectively, our study adds to the growing evidence suggesting that individuals born in the spring and summer have higher cardiovascular mortality than those born in autumn, but conflicts with previous findings on overall mortality. This study supports that the associations of fetal and early life factors with cardiovascular disease mortality could relate to a small but real seasonal effect of fetal or early life factors in later life. Further investigations are required to confirm current findings and uncover mechanisms of seasonal birth month effect in cardiovascular mortality.

We thank all participants and staff of the Nurses' Health Studies for their contributions to this research. We are grateful for help from the following state cancer registries: Alabama, Arizona, Arkansas, California, Colorado, Connecticut, Delaware, Florida, Georgia, Idaho, Illinois, Indiana, lowa, Kentucky, Louisiana, Maine, Maryland, Massachusetts, Michigan, Nebraska, New Hampshire, New Jersey, New York, North Carolina, North Dakota, Ohio, Oklahoma, Oregon, Pennsylvania, Rhode Island, South Carolina, Tennessee, Texas, Virginia, Washington, and Wyoming.

Contributors: ESS and YZ were involved in the study concept and design. YZ, EED, and SS performed the statistical analysis. YZ interpreted data and drafted the manuscript. All authors participated in critical revision of the manuscript for important intellectual content. EED, SS, and FG contributed to administrative, technical, and material support. ESS obtained funding. YZ and ESS had full access to all the data in the study and take responsibility for the integrity of the data and the accuracy of the data analysis. All authors approved the final version of this paper and the authorship list. YZ and ESS are the guarantors. The corresponding author attests that all listed authors meet authorship criteria and that no others meeting the criteria have been omitted.

Funding: This research was supported by grant R01OH009803 from the US Center for Disease Control and Prevention (CDC) and the National Institute for Occupational Safety and Health (NIOSH). The Nurses' Health Study was supported by grant UM1CA186107, and UM1CA176726 from the US National Cancer Institute. The funding sources played no role in the study design, data collection, data analysis, and interpretation of results, or the decisions made in preparation and submission of the article.

Competing interests: All authors have completed the ICMJE uniform disclosure form at www.icmje.org/coi_disclosure.pdf and declare: support from the CDC and NIOSH for the submitted work; no financial relationships with any organizations that might have an interest in the submitted work in the previous three years; no other relationships or activities that could appear to have influenced the submitted work.

Ethical approval: The study protocol was approved by the institutional review board at Brigham and Women's Hospital (1999-P011114). Consent from participants was indicated by the completion and return of the questionnaires.

Data sharing: Data, the statistical code, questionnaires, and technical processes are available from the corresponding author.

The lead author affirms that the manuscript is an honest, accurate, and transparent account of the study being reported; that no important aspects of the study have been omitted; and that any discrepancies from the study as planned (and, if relevant, registered) have been explained.

This is an Open Access article distributed in accordance with the Creative Commons Attribution Non Commercial (CC BY-NC 4.0) license, which permits others to distribute, remix, adapt, build upon this work non-commercially, and license their derivative works on different terms, provided the original work is properly cited and the use is noncommercial. See: http://creativecommons.org/licenses/by-nc/4.0/.

1 Barker DJ. The fetal and infant origins of adult disease. BMJ 1990;301:1111. doi:10.1136/bmj.301.6761.1111

2 Lucas A, Fewtrell MS, Cole TJ. Fetal origins of adult disease-the hypothesis revisited. BMJ 1999:319:245-9. doi:10.1136/ bmj.319.7204.245

3 Gluckman PD, Hanson MA. Living with the past: evolution, development, and patterns of disease. Science 2004;305:1733-6. doi:10.1126/science.1095292

4 Sohn K. The influence of birth season on mortality in the United States. Am J Hum Biol 2016;28:662-70. doi:10.1002/ajhb.22848

Gavrilov LA, Gavrilova NS. Season of birth and exceptional longevity: comparative study of american centenarians, their siblings, and spouses. J Aging Res 2011;2011:104616. doi:10.4061/2011/104616

6 Ueda P, Edstedt Bonamy AK, Granath F, Cnattingius S. Month of birth and mortality in Sweden: a nation-wide population-based cohort study. PLoS One 2013;8:e56425. doi:10.1371/journal.pone.0056425 
7 Ueda P, Edstedt Bonamy AK, Granath F, Cnattingius S. Month of birth and cause-specific mortality between 50 and 80 years: a population-based longitudinal cohort study in Sweden. Eur J Epidemiol 2014;29:89-94. doi:10.1007/s10654-014-9882-7

8 Reffelmann T, Ittermann T, Empen K, Dörr M, Felix SB. Is cardiovascular mortality related to the season of birth?: evidence from more than 6 million cardiovascular deaths between 1992 and 2007. J Am Coll Cardiol 2011;57:887-8. doi:10.1016/j. jacc. 2010.10.021

9 Lerchl A. Month of birth and life expectancy: role of gender and age in a comparative approach. Naturwissenschaften 2004;91:422-5. doi:10.1007/s00114-004-0553-5

10 Doblhammer G, Vaupel JW. Lifespan depends on month of birth. Proc Natl Acad Sci U S A 2001;98:2934-9. doi:10.1073/ pnas.041431898

11 Doblhammer G. Longevity and month of birth: evidence from Austria and Denmark. Demogr Res 1999;1:22. doi:10.4054/ DemRes.1999.1.3

12 Stoupel E, Tamoshiunas A, Radishauskas R, Abramson E, Bernotiene G, Bacevichiene M. Birth month and longevity: birth month of victims of sudden $(S C D, \leq 1 \mathrm{~h})$ and rapid $(R C D, \leq 24 \mathrm{~h})$ cardiac deaths. J Basic Clin Physiol Pharmacol 2013;24:235-9. doi:10.1515/ jbcpp-2012-0075

13 Inoue Y, Stickley A, Yazawa A, Fujiwara T, Kondo K, Kondo N. Month of birth is associated with mortality among older people in Japan: Findings from the JAGES cohort. Chronobiol Int 2016;33:441-7. doi:10.3109/07420528.2016.1152977

14 Muñoz-Tudurí M, García-Moro C. Season of birth affects shortand long-term survival. Am J Phys Anthropol 2008;135:462-8. doi:10.1002/ajpa.20770

15 Villar J, Belizan JM. The timing factor in the pathophysiology of the intrauterine growth retardation syndrome. Obstet Gynecol Surv 1982;37:499-506. doi:10.1097/00006254-198208000-00001

16 Moore SE, Cole TJ, Poskitt EM, et al. Season of birth predicts mortality in rural Gambia. Nature 1997;388:434 doi:10.1038/41245

17 Ward WP. Weight at birth in Vienna, Austria, 1865-1930. Ann Hum Biol 1987;14:495-506. doi:10.1080/03014468700009341

18 Chodick G, Flash S, Deoitch Y, Shalev V. Seasonality in birth weight: review of global patterns and potential causes. Hum Biol 2009;81:463-77. doi:10.3378/027.081.0405

19 Painter RC, Roseboom TJ, Bleker OP. Prenatal exposure to the Dutch famine and disease in later life: an overview. Reprod Toxicol 2005;20:345-52. doi:10.1016/j.reprotox.2005.04.005

20 Bengtsson T, Ohlsson R. Age-specific mortality and short-term changes in the standard of living: Sweden, 1751-1859. Eur J Popul 1985;1:309-26. doi:10.1007/BF01797146

21 Bengtsson T, Lindström M. Airborne infectious diseases during infancy and mortality in later life in southern Sweden, 1766-1894. Int J Epidemiol 2003;32:286-94. doi:10.1093/ije/dyg061

22 Crimmins EM, Finch CE. Infection, inflammation, height, and longevity. Proc Natl Acad Sci U S A 2006;103:498-503. doi:10.1073/pnas.0501470103

23 Sham PC, O'Callaghan E, Takei N, Murray GK, Hare EH, Murray RM Schizophrenia following pre-natal exposure to influenza epidemics between 1939 and 1960. Br J Psychiatry 1992;160:461-6. doi:10.1192/bjp.160.4.461

24 Orme JE. Intelligence, Season of Birth and Climatic Temperature. BrJ Psychol 1963;54:273-6. doi:10.1111/j.2044-8295.1963. tb00882.x

25 Rousham EK, Gracey M. Seasonality of low birthweight in indigenous Australians: an increase in pre-term birth or intrauterine growth retardation?Aust N Z J Public Health 1998;22:669-72. doi:10.1111/ j.1467-842X.1998.tb01467.x

26 Lawlor DA, Davey Smith G, Mitchell R, Ebrahim S. Temperature at birth, coronary heart disease, and insulin resistance: cross sectional analyses of the British women's heart and health study. Heart 2004;90:381-8. doi:10.1136/hrt.2002.009548

27 Bruckner TA, van den Berg GJ, Smith KR, Catalano RA. Ambient temperature during gestation and cold-related adult mortality in a Swedish cohort, 1915-2002. Soc Sci Med 2014;119:191-7. doi:10.1016/j.socscimed.2014.01.049

28 Holick MF. Vitamin D deficiency. N Engl J Med 2007;357:266-81. doi:10.1056/NEJMra070553

29 Theodoratou E, Tzoulaki I, Zgaga L, Ioannidis JP. Vitamin D and multiple health outcomes: umbrella review of systematic reviews and meta-analyses of observational studies and randomised trials. BMJ 2014;348:g2035. doi:10.1136/bmj.g2035

30 Lowell WE, Davis GEJr. The light of life: evidence that the sun modulates human lifespan. Med Hypotheses 2008;70:501-7. doi:10.1016/j.mehy.2007.05.053

31 Buckles KS, Hungerman DM. Season of Birth and Later Outcomes: Old Questions, New Answers. Rev Econ Stat 2013;95:711-24. doi:10.1162/REST_a_00314
32 Kihlbom M, Johansson SE. Month of birth, socioeconomic background and development in Swedish men. J Biosoc Sci 2004; 36:561-71. doi:10.1017/S002193200300628X

33 Bobak M, Gjonca A. The seasonality of live birth is strongly influenced by socio-demographic factors. Hum Reprod 2001;16:1512-7. doi:10.1093/humrep/16.7.1512

34 Smith GD, Hart C, Blane D, Gillis C, Hawthorne V. Lifetime socioeconomic position and mortality: prospective observational study. BMJ 1997;314:547-52. doi:10.1136/bmj.314.7080.547

35 Vaupel JW, Carey JR, Christensen K, et al. Biodemographic trajectories of longevity. Science 1998;280:855-60. doi:10.1126/ science.280.5365.855

36 Belanger CF, Hennekens CH, Rosner B, Speizer FE. The nurses' health study. Am J Nurs 1978;78:1039-40

37 Belanger C, Speizer FE, Hennekens CH, Rosner B, Willett W, Bain C. The nurses' health study: current findings. Am J Nurs 1980;80:1333. doi:10.1097/00000446-198007000-00024

38 Colditz GA, Manson JE, Hankinson SE. The Nurses' Health Study: 20-year contribution to the understanding of health among women. Womens Health 1997;6:49-62. doi:10.1089/jwh.1997.6.49

39 Stampfer MJ, Willett WC, Speizer FE, et al. Test of the National Death Index. Am J Epidemiol 1984;119:837-9. doi:10.1093/ oxfordjournals.aje.a113804

40 Rich-Edwards JW, Corsano KA, Stampfer MJ. Test of the National Death Index and Equifax Nationwide Death Search. Am J Epidemiol 1994:140:1016-9 doi:10.1093/oxfordjournals aje a117191

41 Colditz GA, Martin P, Stampfer MJ, et al. Validation of questionnaire information on risk factors and disease outcomes in a prospective cohort study of women. Am J Epidemiol 1986;123:894-900. doi:10.1093/oxfordjournals.aje.a114319

42 Rimm EB, Stampfer MJ, Colditz GA, Chute CG, Litin LB, Willett WC. Validity of self-reported waist and hip circumferences in men and women. Epidemiology 1990;1:466-73. doi:10.1097/00001648 199011000-00009

43 Wolf AM, Hunter DJ, Colditz GA, et al. Reproducibility and validity of a self-administered physical activity questionnaire. Int Epidemiol 1994;23:991-9. doi:10.1093/ije/23.5.991

44 Benjamin EJ, Muntner P, Alonso A, et al, American Heart Association Council on Epidemiology and Prevention Statistics Committee and Stroke Statistics Subcommittee. Heart Disease and Stroke Statistics-2019 Update: A Report From the American Heart Association. Circulation 2019;139:e56-528. doi:10.1161/CIR.0000000000000659

45 Li L, Boland MR, Miotto R, Tatonetti NP, Dudley JT. Replicating Cardiovascular Condition-Birth Month Associations. Sci Rep 2016;6:33166. doi:10.1038/srep33166

46 Boland MR, Shahn Z, Madigan D, Hripcsak G, Tatonetti NP. Birth month affects lifetime disease risk: a phenome-wide method. J Am Med Inform Assoc 2015;22:1042-53. doi:10.1093/jamia/ocv046

47 Poltavskiy E, Spence JD, Kim J, Bang H. Birth Month and Cardiovascular Disease Risk Association: Is meaningfulness in the eye of the beholder?Online J Public Health Inform 2016;8:e186. doi:10.5210/ojphi.v8i2.6643

48 Barker DI. The developmental origins of adult disease. Eur J Epidemiol 2003;18:733-6. doi:10.1023/A:1025388901248

49 Marciniak A, Patro-Matysza J, Kimber-Trojnar Ż, Marciniak B, Oleszczuk J, Leszczyńska-Gorzelak B. Fetal programming of the metabolic syndrome. Taiwan J Obstet Gynecol 2017;56:133-8. doi:10.1016/j. tjog.2017.01.001

50 Roseboom TJ. Undernutrition during fetal life and the risk of cardiovascular disease in adulthood. Future Cardiol 2012;8:5-7. doi:10.2217/fca.11.86

51 Mazumder B, Almond D, Park K, Crimmins EM, Finch CE. Lingering prenatal effects of the 1918 influenza pandemic on cardiovascular disease. J Dev Orig Health Dis 2010;1:26-34. doi:10.1017/ S2040174409990031

52 Kaplan GA, Salonen JT. Socioeconomic conditions in childhood and ischaemic heart disease during middle age. BMJ 1990;301:1121-3. doi:10.1136/bmj.301.6761.1121

53 Kasahara AK, Singh RJ, Noymer A. Vitamin D (250HD) Serum Seasonality in the United States. PloS One 2013:8:e65785. doi:10.1371/journal.pone.0065785

54 Lv J, Yu C, Guo Y, et al, China Kadoorie Biobank collaborative group. The associations of month of birth with body mass index, waist circumference, and leg length: findings from the China Kadoorie Biobank of 0.5 million adults. J Epidemiol 2015;25:221-30. doi:10.2188/jea.JE20140154

55 Day FR, Forouhi NG, Ong KK, Perry JR. Season of birth is associated with birth weight, pubertal timing, adult body size and educationa attainment: a UK Biobank study. Heliyon 2015;1:e00031. doi:10.1016/j.heliyon.2015.e00031

Infographic: Monthly and seasonal hazard ratios for cardiovascular death from fully adjusted Cox models 


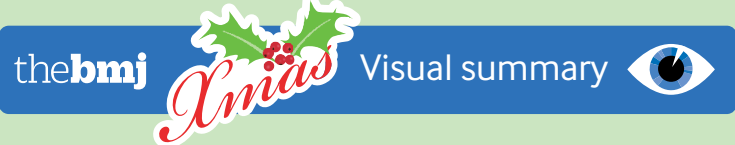

Consistent patterns have been observed linking birth in the spring and summer with higher risk of cardiovascular disease in the northern hemisphere, with the reverse pattern south of the equator. Authors Zhang and colleagues observed this pattern in the Nurses' Health Study longitudinal cohort, a group of 121700 female registered nurses recruited in the United States in 1976.

The diagram below shows the results of the authors' fully adjusted Cox model, providing monthly and seasonal hazard ratios for cardiovascular disease death. They did not observe a similar pattern for overall mortality. Full results can be seen on bmj.com at the link below.

\section{Birth timing and heart disease}

Association between birth month and season with risk of developing cardiovascular disease

\section{Potential mechanisms}

The reasons for variations in risk of cardiovascular disease with different birth timings are not well understood, but could include prenatal and early postnatal exposures such as:

\section{seasonal fluctuations in nutrition availability}

infections and inflammatory causes climatic temperature

air pollution levels amount of sunlight available
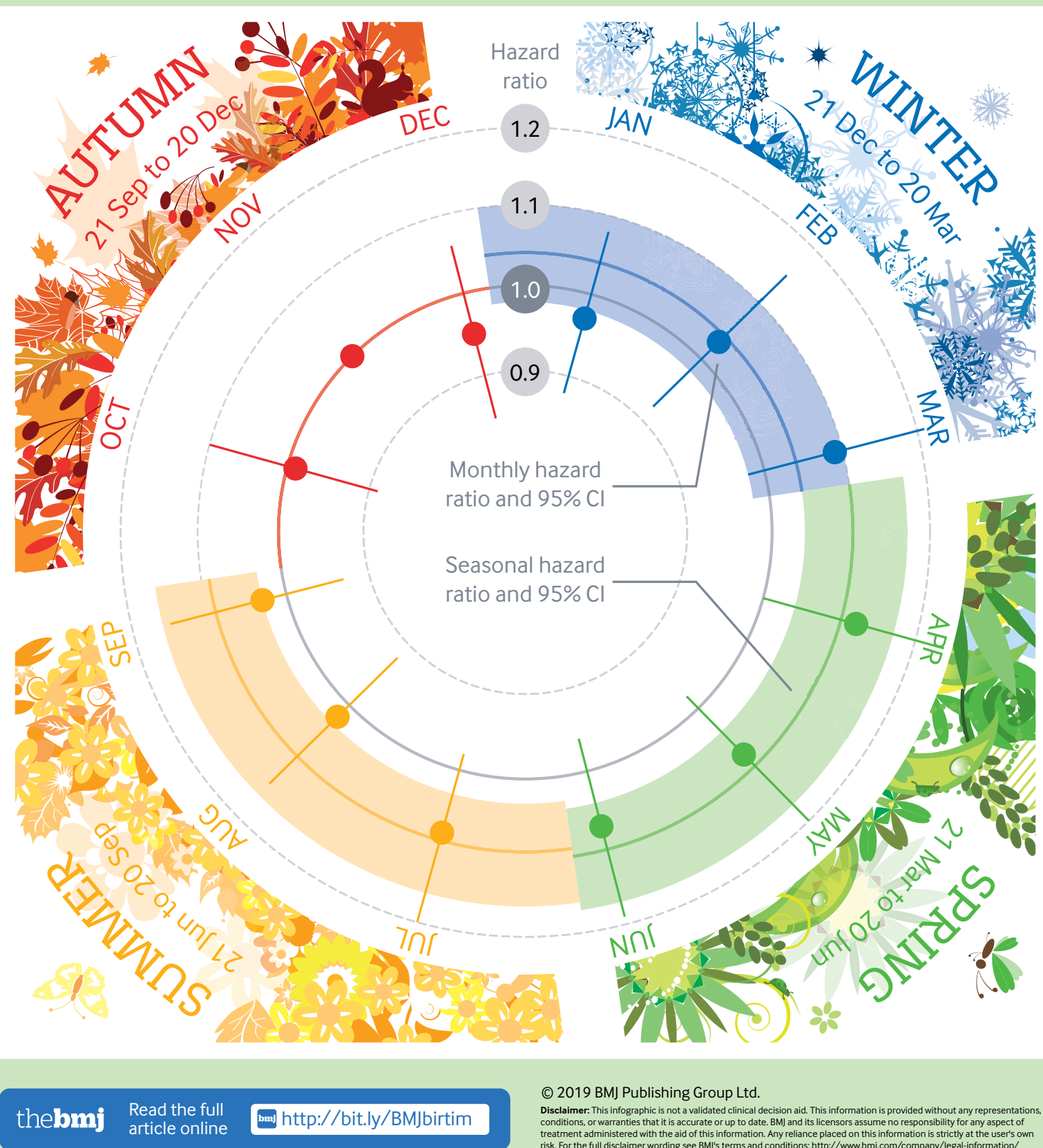

C) 2019 BMJ Publishing Group Ltd.

Disclaimer: This infographic is not a validated clinical decision aid. This information is provided without any representations.

reatment administered with the aid of this information. Any reliance placed on this information is strictly at the user's ow

(1) See more visual
summaries http://www.bmj.com/infographics 